\title{
Identificación de las variables que afectan a la percepción de las transferencias en los viajes multietapa: focus groups en Madrid y Vitoria
}

\author{
Fernando Herrero Ruiz \\ Investigador predoctoral, TRANSyT-UPM, España \\ Rocío Cascajo Jiménez \\ Investigadora, TRANSyT-UPM, España \\ Andrés Monzón de Cáceres \\ Catedrático de Transportes, UPM, España
}

\begin{abstract}
RESUMEN
Las transferencias constituyen una etapa esencial en los viajes multimodales, que deben mejorase para aumentar la eficiencia de las redes de transporte público. Los usuarios tienden a percibir estas transferencias como una penalización dentro de su cadena de viaje. Por ello, es importante identificar qué variables influyen en la percepción de las transferencias así como cuantificar su efecto sobre este fenómeno.
\end{abstract}

Existe amplia literatura referente a la cuantificación del efecto de las variables que influyen sobre la penalización de las transferencias; sin embargo, estos estudios no especifican cómo se han elegido esas variables o por qué. Esta investigación pretende identificar las variables que afectan a la penalización de las transferencias de modo que los responsables de las políticas de transporte puedan concentrarse en ellas y conseguir así un uso más eficiente de los fondos públicos.

Esta investigación se enmarca en el proyecto de I+D+i TRANSFER (Penalización de las transferencias en viajes Multietapa: percepción del usuario) que ha permitido identificar un total de 47 variables que influyen en las transferencias, y bajo el que se han llevado a cabo unos estudios cualitativos en dos ciudades españolas, Vitoria y Madrid, consistentes en varios grupos de discusión estratificados por edad. A partir de ellos, se han detectado cuáles son los factores más influyentes en la percepción de la penalización de la transferencia. Finalmente se ofrecen algunas sugerencias y recomendaciones para los decisores políticos.

\section{INTRODUCCIÓN}

En la actualidad, la congestión en las grandes ciudades está haciendo que las redes de transporte público sean menos eficientes. Este es un problema cada vez mayor para los responsables políticos y los operadores, que deben tratar de ofrecer un servicio de transporte público de alta calidad en un contexto donde la eficiencia operativa está siendo cada vez más importante. Es, por tanto, esencial promover el uso del transporte público asegurando un viaje puerta a puerta cómodo y rápido.

Los viajes puerta a puerta en transporte público tiene una gran desventaja para el usuario en comparación con los viajes puerta a puerta en coche: está compuesto por diferentes fases que incluyen acceso y egreso a la parada, tiempo de espera al vehículo, tiempo en el/los vehículos y tiempo de caminata, en el caso de hacer transbordos. A pesar de que todos estos pasos son penalizados por los usuarios, no todos son percibidos por igual, ya que la percepción que los usuarios tienen del tiempo de viaje puede diferir del tiempo real de viaje (Hess et al., 2004; Iseki y Taylor, 2009; Moreau, 1992). Es por ello que algunos de estos 
pasos, tales como los transbordos, sean percibidos como una molestia mayor para los usuarios del transporte.

Los transbordos representan una interrupción en el viaje en transporte público para hacer un cambio de línea o un cambio de modo. El hecho de que los transbordos en los viajes multimodales suponen una pérdida de utilidad ha quedado claro desde hace muchos años (Wardman, 2001), aunque la cuestión de cómo evaluar su valor es una cuestión más discutible. El concepto de transferencia varía mucho con los autores. Tradicionalmente no se ha diferenciado entre el tiempo de espera y de caminata. Guo (2003) define la penalización por transferencia como la desutilidad de una opción con transbordo en comparación con una opción en la que no hay transbordo, mientras que Horowitz y Zlosel (1981) la consideran como el coste extra que supone el esfuerzo adicional necesario para hacer las conexiones. Otro enfoque, que ha surgido en los últimos años, sugiere que la penalización por transferencia no es constante, sino que depende de una serie de factores. Currie (2005) define la penalización por transferencia como el valor percibido de hacer una transferencia entre dos vehículos de transporte público, además del tiempo dedicado a caminar y a esperar necesario para completar la transferencia. Se introduce la penalización por transferencia $\left(\mathrm{P}_{\mathrm{T}}\right)$ que Iseki y Taylor (2009) definen:

donde:

$$
P_{T}=\left(\operatorname{Cam}_{t T} \cdot \operatorname{Cam}_{V}\right)+\left(E s p_{t T} \cdot E s p_{V}\right)+P T_{n}
$$

$P_{T:}$ penalización total por transferencia

Cam $_{t}$ : tiempo (minutos) de caminata en la transferencia

Cam $_{v}$ : valoración del tiempo de caminata por parte del pasajero

Esp tr: tiempo de espera en la transferencia

Esp $p_{v}$ : valoración del tiempo de espera por parte del pasajero

$P T_{n}$ : penalización por transferencia, excluyendo caminata y espera en la transferencia (penalización pura)

Según la expresión (1), la penalización por transferencia tiene tres componentes: una penalización de tiempo de caminata y su percepción; una penalización de tiempo de espera y su percepción; y una penalización de transferencia pura, independiente de la caminata y el tiempo de espera, pero que depende de otros factores relacionados con la seguridad, la comodidad, el clima o las condiciones personales. La penalización por transferencia pura se denomina genéricamente "disrupción". Para poder entender la penalización que experimentan los usuarios del transporte público durante una transferencia, es necesario identificar las variables que afectan a cada uno de estos componentes por separado.

Este artículo se centra en la pérdida de utilidad o penalización asociada a las transferencias en los viajes multimodales en transporte público. El objetivo es identificar las variables que afectan a la disrupción en estos viajes a través de un estudio cualitativo basado en grupos de discusión (focus groups), para en una futura investigación poder realizar encuestas de preferencias declaradas y así medir la desutilidad asociada a las transferencias.

\section{VARIABLES QUE AFECTAN LA PENALIZACIÓN POR TRANSFERENCIA}

\subsection{Tiempo de caminata y tiempo de espera en las transferencias}

El tiempo de caminata a pie es el tiempo desde la plataforma de transferencia de llegada a la plataforma de salida del siguiente vehículo (Guo, 2003). El tiempo de espera en la transferencia se refiere al tiempo que un viajero espera en la plataforma para montarse en el siguiente vehículo. Ambos tiempos de caminata y de espera son fácilmente medibles. Así, el tiempo de espera depende directamente de la frecuencia de los servicios de conexión, mientras que el tiempo de caminata depende directamente de la distancia y de la velocidad 
de la marcha para llegar a la plataforma de conexión. A su vez, la velocidad de caminata también está influenciada por la existencia de escaleras mecánicas o ascensores, y por la condición física del usuario. Sin embargo, la percepción de estos tiempos depende de muchos factores que son internos o externos al usuario (Dziekan, 2008; Iseki y Taylor, 2009). Según Iseki y Taylor (2009) y Wardman y Hine (2000), las variables de percepción se pueden clasificar en función de si constituyen factores internos o externos.

- Factores internos: son variables que dependen por completo de la persona, sus creencias y preferencias personales. Este grupo de variables incluye la familiaridad con la ciudad, con la red de transporte público o la línea/estación, la condición física del usuario, si el usuario va con prisa, etc. Muchas de estas variables están relacionadas con la capacidad de control que experimenta el usuario. Así, los usuarios manifiestan una mayor intención de tomar rutas que implican transferencias cuando tienen un mayor control sobre la situación (Chowdhury y Ceder, 2013a).

- Factores externos: son variables que no dependen de la persona. La frecuencia del servicio, las condiciones climáticas, y la seguridad han sido identificados en la literatura como factores externos que influyen en la percepción del tiempo de espera (Dziekan y Vermeulen, 2006; Dziekan, 2008; Iseki y Taylor, 2009). La inactividad también surgió como un factor externo relevante para este componente, ya que muchos estudios indican que se penaliza más el tiempo de espera cuanto más improductivo es (Dziekan y Vermeulen, 2006; Iseki y Taylor, 2009; Moreau, 1992). Esto también puede explicar las diferencias observadas entre la percepción del tiempo de espera y la del tiempo de caminata. La disponibilidad de información, ya sea información estática o en tiempo real en los puntos de conexión o información a través de apps, y la fiabilidad del servicio también se identificaron en la literatura como factores externos relevantes en la percepción del tiempo de espera y de caminata (Hess et al., 2004, Iseki y Taylor, 2009). Buenos niveles de estas dos variables permiten a los usuarios reducir su tiempo percibido a la vez que aumenta su control sobre la situación y, por tanto, su intención de hacer transferencias (Chowdhury y Ceder, 2013A, 2013b).

\subsection{Penalización por transferencia pura}

Pocos estudios se centran en analizar la penalización pura por transferencia, y por ello es complicado identificar claramente las variables que influyen en ella. Sin embargo, existen investigaciones que mencionan algunas de estas variables (Currie, 2005; Iseki y Taylor, 2009; Liu et al., 1997; Navarrete y Ortúzar, 2013; Palmer et al, 2011; Wardman, 2001; Wardman et al, 2001). La mayoría señala a los modos de transporte que participan en la transferencia como una variable significativa (Currie, 2005; Iseki y Taylor, 2009; Liu et al., 1997; Navarrete y Ortúzar, 2013; Palmer et al, 2011; Wardman et al, 2001); en general, las transferencias metro-metro se prefieren a las transferencias bus-bus.

Wardman (2001) indica que la falta de familiaridad con una estación o con un transbordo, las frecuencias bajas o la escasa fiabilidad penalizan las transferencias, independientemente del tiempo de espera o de caminata. En esta línea, Wardman et al. (2001) y Guo y Wilson (2004) sugieren una alta variabilidad en la penalización por transferencia dependiendo de la estación donde se produce. Esto sugiere la importancia de los factores ambientales, tales como la iluminación, la posibilidad de resguardarse, la información (en tiempo real o estática), el confort, la seguridad, existencia de aseos, etc. Algunos estudios (Douglas y Jones, 2013; Raveau et al, 2014) han demostrado la importancia que tiene la diferencia de nivel en una transferencia en la penalización de la misma, especialmente si no existen 
escaleras mecánicas o ascensores.

Es importante tener en cuenta que ciertas variables aparecen en más de una componente de la penalización global por transferencia. Por ejemplo, una transferencia que tiene lugar a diferentes niveles afecta al tiempo real de caminata -ya que la gente camina más despacio en las escaleras-; a la percepción del tiempo de caminata -ya que se trata de un esfuerzo adicional-; y, por último, a la penalización pura por transferencia -ya que las escaleras psicológicamente disuaden al usuario de realizar la transferencia.

Las principales variables que afectan a la penalización por transferencia identificadas en la literatura son: tiempo de espera, tiempo de caminata, el confort, la seguridad y la información. Esto sugiere que estas variables recurrentes pueden constituir los principales componentes de la percepción de las transferencias de los usuarios. Sin embargo, sólo unos pocos estudios consideran la penalización pura por transferencia de manera aislada al tiempo de espera y de caminata (Douglas y Jones, 2013; Wardman y Hine, 2000). Iseki y Taylor (2009) han demostrado que las penalizaciones por transferencia varían entre ciudades, apuntando la necesidad de realizar estudios para verificar este hecho aplicando la misma metodología a diferentes ciudades.

\section{METODOLOGÍA}

\subsection{Aproximaciones al problema en la literatura}

Existen pocos estudios que identifiquen las variables más importantes que intervienen en la penalización por transferencia. Entre ellos, se han detectado dos enfoques:

El primero consiste en la realización de encuestas SP a los usuarios del transporte público en varias estaciones de intercambio (Ceder et al, 2013; Chowdhury y Ceder, 2013a, 2013b; Chowdhury et al, 2014; Douglas y Jones, 2013; Liu et al, 1997; Navarrete y Ortúzar, 2013; Schakenbos, 2014). Los datos obtenidos de estos estudios se analizan posteriormente utilizando diferentes modelos estadísticos, por lo general después de una especificación logit, para revelar qué variables son significativas.

El segundo enfoque comprende algún tipo de revisión de estudios previos sobre el tema para examinar sus datos y conclusiones. Este es el caso de Iseki y Taylor (2009), que hacen una extensa revisión de la literatura, y Wardman y Hine (2000), que llevan a cabo un metaanálisis de estudios previos sobre el tema. La segunda metodología tiene la ventaja de proporcionar un análisis más detallado que los estudios aislados en los que se basan, mediante la explotación de sus pruebas en su conjunto (Abrantes y Wardman, 2011).

Estos dos enfoques se basan en metodologías cuantitativas para identificar las variables que afectan a la penalización por transferencia.

A la vista de la falta de estudios cualitativos que capturen los sentimientos y las intenciones de los usuarios hacia las transferencias, y permitan identificar las variables que más les influyen en la percepción de las transferencias, se decidió aplicar una metodología cualitativa conocida como grupo de discusión (GD). De acuerdo con Greenbaum (1999), los GD son una técnica de investigación cualitativa que consiste en pequeños grupos (4-10 personas) reclutados para llevar a cabo un debate sobre un tema específico dirigido por un moderador experto. La idea principal es la interacción entre los participantes. Los GD tienen algunas ventajas sobre el análisis cuantitativo: es un proceso dinámico en el que el grupo tiene en cuenta lo que se ha aprendido de las intervenciones anteriores, y permite a los investigadores obtener las actitudes y sentimientos de entender el "por qué" de las decisiones de los usuarios 
(Carreira et al, 2013; Greenbaum, 1999), ya que los participantes no están limitados por la estructura fija de los cuestionarios formales (Hine y Scott, 2000). No se trata de una metodología innovadora, que ya se ha utilizado en otras ocasiones en estudios de transporte para obtener datos cualitativos directamente de los usuarios (Carreira et al, 2013; Hine y Scott, 2000; Ibeas et al., 2011). Con todo, esta metodología no se ha aplicado convencionalmente para identificar las variables que afectan a la penalización por transferencia. Una excepción es la investigación de Navarrete (2010), en la que el autor utiliza GD para identificar las variables relevantes para una encuesta SP sobre la penalización de las transferencias en Santiago de Chile.

\subsection{Descripción de los casos de estudio}

Se han seleccionado dos ciudades españolas para identificar las variables que más influyen en la percepción de las transferencias a través de grupos focales: Vitoria y Madrid.

Vitoria es una ciudad de tamaño medio, con 243.918 habitantes (INE, 2015), que fue Capital Verde Europea en 2012. Su sistema de transporte consiste en una red de autobuses con diez líneas y una red de tranvía con dos líneas. Una característica importante de esta ciudad es su tamaño compacto, ya que la mayoría de la población se concentra en una radio de $3 \mathrm{~km}$ del centro de la ciudad. Esto da lugar a la siguiente distribución modal (2014): 24\% en coche, $53,5 \%$ a pie, $13,4 \%$ en bicicleta y $7,9 \%$ en transporte público. Tan solo el $0,45 \%$ de viajes son intermodales (TRANSyT, 2015). El 40,7\% de los desplazamientos es de menos de 10 minutos, el 74,8\% de menos de 20 y casi el $90 \%$ de menos de 30 minutos. Vitoria tiene inviernos fríos con precipitaciones frecuentes, a veces en forma de nieve. Se eligió Vitoria como caso de estudio debido a sus patrones de movilidad sostenibles, y porque ha cambiado su red de transporte en los últimos años hacia una red más eficiente con menos líneas y servicios más frecuentes, pero con una mayor necesidad de transbordar entre las líneas.

Madrid es la ciudad más habitada de España, con 3,14 millones de habitantes y casi 6,5 millones en su área metropolitana (INE, 2015). Su red de transporte consta de 204 líneas de autobuses urbanos, 109 líneas de autobuses interurbanos, 12 líneas de metro, 4 líneas de metro ligero y 9 líneas de Cercanías. Esta densa red de transportes produce una distribución modal equilibrada: $38,9 \%$ viajes se realizan en coche, el $28,4 \%$ en el transporte público, el $29,9 \%$ a pie y en bicicleta $0,5 \%$; el $9 \%$ de los viajes son intermodales (Encuesta Sintética de Movilidad, 2014). El clima de Madrid es frío en invierno y caluroso en verano, con precipitaciones concentradas en primavera y otoño, poco frecuentes en verano. Se eligió Madrid como caso de estudio debido a su red de transporte público densa, con varios modos, diferentes tipos de puntos de intercambio y la posibilidad de utilizar diferentes rutas para hacer el mismo viaje.

Estos dos casos de estudio permiten la comparación de las variables en dos ciudades de diferentes tamaños con diferentes redes de transporte público y patrones de movilidad.

\section{GRUPOS DE DISCUSIÓN}

\subsection{Reclutamiento, selección de participantes y realización de los grupos de discusión}

Se hizo un reclutamiento separado en cada ciudad. Debido a las diferencias encontradas en la literatura sobre las limitaciones físicas de las personas mayores, se decidió llevar a cabo los diferentes grupos por edad (menores o mayores de 65 años). Esta agrupación por edad también se aplicó en Navarrete (2010), aunque con diferentes rangos de edad.

En Vitoria, se realizaron dos grupos de discusión: uno de menores de 65 años, en el que 
participaron 5 personas), y otro de mayores de 65 años, en le que participaron 4 personas. En Madrid, además de estos dos grupos, se formó un tercer grupo de estudiantes que sirvió como grupo piloto. 8 participantes fueron elegidos, asegurando que género y edad fueran representados de manera uniforme. El grupo de menores de 65 años fue seleccionado entre empleados de la Universidad Politécnica de Madrid, y en él participaron 7 personas. Para el grupo de más de 65 años, un total de 5 personas asistieron finalmente a la reunión.

Todas las sesiones se llevaron a cabo de una manera similar, y fueron conducidas por un psicólogo. La duración de las sesiones fue de aproximadamente una hora y media. Al comienzo de cada una, se informó a los participantes del propósito y la confidencialidad de los datos y las declaraciones dentro del grupo. Luego se les explicó del funcionamiento del GD. Siguiendo las recomendaciones de Greenbaum (1999), los investigadores sólo intervinieron para fomentar la conversación y para asegurarse de que todos los participantes participaban.

\subsection{Resultados de los grupos de discusión}

La variable principal que surge en todos los grupos de discusión es el tiempo, en sus diferentes componentes (tiempo de caminata, de espera, de transferencia, o el tiempo total de viaje). El tiempo fue mencionado por el $79 \%$ de los participantes, siendo algo más importante en Vitoria que en Madrid, probablemente debido a los menores tiempos de viaje a pie. Esto es coherente con la literatura consultada (Iseki y Taylor, 2009; Wardman y Hine, 2000). El valor del tiempo también parece ser menos importante en los ancianos que los jóvenes.

Las aplicaciones móviles e información en tiempo real quedaron en segundo lugar en todos los grupos. Mientras que los jóvenes prefieren consultar información en tiempo real a través de apps (incluso decidir cuál es la estación para transbordar al comprobar el tiempo de espera real de los servicios de conexión en sus teléfonos móviles), los participantes mayores prefieren la información en tiempo real proporcionada en los paneles dinámicos en las paradas. En los últimos años, muchos estudios han examinado los beneficios de la información en tiempo real a los pasajeros (Monzón et al, 2013; Tang y Thakuriah, 2012). Algunos de ellos son que proporcionan una mejor utilización del tiempo de espera, unos efectos psicológicos positivos (como la reducción de la incertidumbre), mayor sensación de seguridad, una sensación de confianza en el sistema de transporte público, una mayor facilidad de uso, y una mayor satisfacción de los pasajeros.

La frecuencia del servicio y la fiabilidad también han sido variables relevantes para las transferencias. Estas variables son más importantes en Vitoria (mencionadas por el $67 \%$ de los participantes) que en Madrid (mencionadas por el 50\% de los participantes). Esto puede deberse a que las frecuencias y distancias en Vitoria son más bajas, y las personas son más sensible a este tema.

Otra variable resaltada en la literatura y que surgió en los grupos es el modo de transporte utilizado en el viaje. Esto fue mencionado por el $48 \%$ de los participantes, y era más importante en Madrid (55\%) que en Vitoria (33\%), probablemente debido a la mayor variedad de modos de transporte.

La caminata durante la transferencia fue mencionada por el $52 \%$ de los participantes, con el mismo porcentaje en ambas ciudades. Sin embargo, esta variable parece ser percibida de manera diferente por los grupos de edad. Mientras que sólo fue mencionada por el $38 \%$ de 
los estudiantes, el porcentaje aumentó en el grupo de 18-65 (42\%) y fue muy importante en los ancianos, cuando fue mencionado por el $78 \%$ de los participantes. Lo mismo ocurre con la familiaridad del usuario con la estación o ruta. A pesar de que se menciona en un porcentaje similar en ambas ciudades (45\% en Madrid, 56\% en Vitoria), las diferencias significativas se observaron entre los grupos de edad: fue mencionado por sólo el $38 \%$ de los estudiantes, pero un $78 \%$ de las personas mayores en ambos grupos.

Algunas variables han sido más importantes en Madrid que en Vitoria. La aglomeración fue mencionada por el 60\% de los participantes en Madrid; diferentes niveles de profundidad en las transferencias, por el 55\%; información genérica, por el $45 \%$ y la hora del día, por el $40 \%$. Esto puede deberse a que estos fenómenos son característicos de Madrid, que tiene varias estaciones e intercambiadores muy transitados, una red compleja, y algunas estaciones de metro muy profundas. También vale la pena señalar que, aunque la aglomeración, la información y las transferencias entre diferentes niveles aparecieron en la literatura, una nueva variable surgió en los grupos de discusión: una penalización psicológica debida a la profundidad de la estación.

Otras variables resultaron ser más relevante en Vitoria que en Madrid. Por ejemplo, la coordinación entre los modos y servicios fue mencionada por el $56 \%$ de los participantes en Vitoria. Muchos de ellos se quejaron de la falta de coordinación, sobre todo en los servicios de autobús.

El tiempo atmosférico fue otra variable relevante en Vitoria, que se menciona en un $44 \%$. Esto es debido al clima frío y lluvioso de la ciudad, lo que significa que la gente prefiere esperar al próximo autobús a caminar bajo la lluvia o la nieve. Por lo tanto, las transferencias son preferidas cuando llueve o nieva.

Por último, se encontraron dos variables que no aparecen en la literatura, y que están relacionadas con la penalización pura. La primera es el esfuerzo mental extra requerido por los pasajeros al hacer una transferencia y la necesidad de permanecer alerta durante todo el viaje con el fin de no perder su parada de transferencia, que surgió en todos los GD, excepto los de más de 65 años. La segunda se refiere al uso que se hace del tiempo en el vehículo, especialmente en viajes más largos cuando el tiempo se puede utilizar para leer, escuchar música o incluso dormir. Las transferencias fueron vistas como una interrupción en esta actividad asociada al hecho de transferir.

Una vez identificadas las variables, el paso siguiente debería implicar la cuantificación del efecto de cada una de estas variables sobre la penalización experimentada por el usuario cuando transfiere.

\section{CONCLUSIONES}

El objetivo de este trabajo es identificar las variables que afectan a la percepción de los transbordos en los desplazamientos urbanos multimodales para poder mejorar la experiencia de viaje para los usuarios del transporte público. Para ello se ha llevado a cabo un estudio cualitativo, consistente en varios grupos de discusión, en dos ciudades españolas. Tanto la literatura como los resultados de los GD destacan que una reducción en el tiempo de caminata y de espera en las transferencias es la medida más eficaz para disminuir la penalización de las transferencias. Una mejora de las frecuencias disminuiría el tiempo de espera, mientras que una mejora en las condiciones de espera (asientos, temperatura, iluminación, seguridad, etc.) contribuiría a mejorar la percepción de los usuarios. Los nuevos 
diseños de estaciones deben tener como objetivo reducir las distancias entre paradas/estaciones, e introducir pasillos rodantes para reducir los tiempos de caminata. Además, los responsables políticos deben concentrar sus esfuerzos en proporcionar información en tiempo real, ya sea a través de paneles dinámicos en las paradas o por medio de apps, siendo esta última opción preferida sólo por los participantes más jóvenes.

Las características de la ciudad, como el tamaño, las pautas de movilidad y el diseño de la red de transporte público no deben pasarse por alto cuando se trata de mejorar la percepción de las transferencias en los viajes urbanos multimodales. Así, debería mejorarse la frecuencia y la fiabilidad del servicio en pequeñas ciudades como Vitoria, con distancias menores, y diseñar unas estaciones menos profundas y mejorar la señalización en ciudades más pobladas como Madrid, con las redes de transporte público complejas.

Finalmente, hay que señalar que hay una resistencia a transbordar causada por el esfuerzo mental que aparentemente experimentan los usuarios en respuesta a la interrupción de cualquier actividad que puedan desarrollar en el interior del vehículo. Sin embargo, hay una gran cantidad de medidas que los responsables políticos pueden tomar para reducir esta penalización al mínimo y mejorar así la disposición de los usuarios a utilizar el TP.

\section{REFERENCIAS}

Abrantes, P. A., \& Wardman, M. R. (2011). Meta-analysis of UK values of travel time: An update. Transportation Research Part A: Policy and Practice, 45(1), 1-17.

Carreira, R., Patrício, L., Natal Jorge, R., Magee, C., Van Eikema Hommes, Q. (2013). Towards a holistic approach to the travel experience: A qualitative study of bus transportation. Transp. Policy 25, 233-243.

Ceder, A., Hadas, Y., McIvor, M., Ang, A. (2013). Transfer Synchronization of Public Transport Networks. Transp. Res. Rec. J. Transp. Res. Board 2350, 9-16.

Chowdhury, S., \& Ceder, A. (2013a). A psychological investigation on public-transport users' intention to use routes with transfers. International Journal of Transportation, 1(1), $1-20$.

Chowdhury, S., \& Ceder, A. (2013b). The effect of interchange attributes on public-transport users' intention to use routes involving transfers. Psychology and Behavioral Sciences, 2(1), 5-13.

Chowdhury, S., Ceder, A., Sachdeva, R. (2014). The effects of planned and unplanned transfers on public transport users' perception of transfer routes. Transp. Plan. Technol. $37,154-168$.

Currie, G. (2005). The Demand Performance of Bus Rapid Transit. J. Public Transp. 8, 4155.

Douglas, N.J., Jones, M. (2013). Estimating Transfer Penalties and Standardised Income Values of Time by Stated Preference Survey. Aust. Transp. Res. Forum 1-21.

Dziekan, K. (2008). Ease-of-use in public transportation: a user perspective on information and orientation aspects. Dr. Thesis. Royal Institute of Technology (KTH).

Dziekan, K., Vermeulen, A. (2006). Psychological effects of and design preferences for realtime information displays. J. Public Transp. 9, 1-19.

Greenbaum, T.L. (1999). Moderating Focus Groups: A Practical Guide for Group Facilitation. SAGE publications.

Guo, Z. (2003). Assessment of the Transfer Penalty for Transit Trips in Downtown Boston. A GIS-based Disaggregate Modeling Approach. Master Thesis. Massachusetts Institute of Technology.

Guo, Z., \& Wilson, N. (2004). Assessment of the transfer penalty for transit trips geographic 
information system-based disaggregate modeling approach. Transportation Research Record: Journal of the Transportation Research Board, (1872), 10-18.

Hess, D.B., Brown, J., Shoup, D. (2004). Waiting for the Bus. J. Public Transp. 7, 67-84.

Hine, J., Scott, J. (2000). Seamless, accessible travel: Users' views of the public transport journey and interchange. Transp. Policy 7, 217-226.

Horowitz, A. J., \& Zlosel, D. J. (1981). Transfer penalties: Another look at transit riders' reluctance to transfer. Transportation, 10(3), 279-282.

Ibeas, A., Dell'Olio, L., Montequín, R.B. (2011). Citizen involvement in promoting sustainable mobility. J. Transp. Geogr. 19, 475-487.

Instituto Nacional de Estadística, 2015. Estadística del Padrón Continuo a 1 de enero de 2015.

Iseki, H., Taylor, B.D. (2009). Not All Transfers Are Created Equal: Towards a Framework Relating Transfer Connectivity to Travel Behaviour. Transp. Rev. 29, 777-800.

Liu, R., Pendyala, R., \& Polzin, S. (1997). Assessment of intermodal transfer penalties using stated preference data. Journal of the Transportation Research Board, (1607), 74-80.

Monzón, A., Hernández, S., Cascajo, R. (2013). Quality of Bus Services Performance: Benefits of Real Time Passenger Information Systems. Transp. Telecommun. 14 (2), 155 166.

Moreau, A. (1992). Public transport waiting times as experienced by customers. Public Transp. Int. 41 (3).

Navarrete, F.J. (2010). Valoración Subjetiva del Transbordo en Transporte Público: Caso Santiago de Chile. Dr. Thesis. Pontificia Universidad Católica de Chile.

Navarrete, F.J., Ortúzar, J.D. (2013). Subjective valuation of the transit transfer experience: The case of Santiago de Chile. Transp. Policy 25, 138-147.

Palmer, D., James, C., Jones, M. (2011). Door to Door Journeys. Report produced by Transport Research Laboratory for Campaign for better Transport.

Raveau, S., Guo, Z., Muñoz, J.C., Wilson, N.H.M. (2014). A behavioural comparison of route choice on metro networks: Time, transfers, crowding, topology and sociodemographics. Transp. Res. Part A Policy Pract. 66, 185-195.

Schakenbos, R. (2014). Valuation of a transfer in a multimodal PTt trip. Master thesis. University of Twente.

Shires, J.D., de Jong, G.C. (2009). An international meta-analysis of values of travel time savings. Eval. Program Plann. 32, 315-325.

Tang, L., \& Thakuriah, P. V. (2012). Ridership effects of real-time bus information system: A case study in the City of Chicago. Transp. Res. Part C Emerg. Technol. 22, 146-161.

TRANSyT - Centro de Investigación del Transporte UPM (2015). Encuesta de Movilidad de Vitoria-Gasteiz 2014. Análisis de resultados. Centro de Estudios Ambientales. Ayuntamiento de Vitoria-Gasteiz.

Wardman, M. (2001). A review of British evidence on time and service quality valuations. Transp. Res. Part E Logist. Transp. Rev. 37, 107-128.

Wardman, M., Hine, J. (2000). Costs of Interchange: A Review of the Literature. Inst. Transp. Stud. Univ. Leeds, Work. Pap. 546.

Wardman, M., Hine, J., Stradling, S. (2001). Interchange and Travel Choice. Edinburgh Scottish Exec. Cent. Res. Unit 1. 